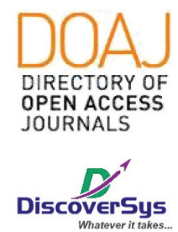

Published by DiscoverSys

\section{A-41 years old male with alcoholic cardiomyopathy at Tabanan General Hospital, Bali-Indonesia: a case report}

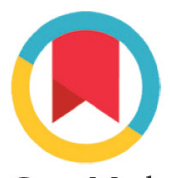

CrossMark

\author{
Gede Wikananda ${ }^{1 *}$, Eka Ariawan², Ary Priadnyana ${ }^{3}$
}

\section{ABSTRACT}

Background: Alcoholic cardiomyopathy is a form of toxic cardiomyopathy which develops as a result of long-standing chronic ingestion of alcohol. Its clinical findings share similar features to other forms of dilated cardiomyopathy. Diagnosis can be made based on the history of long term alcohol abuse, clinical findings, and supporting diagnostic studies. This case report aims to overview alcoholic cardiomyopathy; from establishing the initial diagnosis to the treatment given for the condition.

Case Description: A 41 years old male came to the emergency department unit with shortness of breath since last few days. Shortness of breath was said to be getting worse over time and progressed to breathlessness at rest. Other complaints were palpitations, fatigue, and long-standing cough. The patient was found to have a history of drinking alcohol in large quantities since 27 years ago. The blood pressure was $110 / 70 \mathrm{~mm} \mathrm{Hg}$, pulse rate 105 beats/min, and respiratory rate was 20 breaths $/ \mathrm{min}$. Slight edemas on both legs were also found. From auscultation examination, bibasilar crepitation on both lungs was found. Cardiac examination showed systolic murmur of mitral regurgitation (MR) and tricuspid regurgitation (TR). On supporting investigation, a chest $x$-ray showed cardiomegaly (cardiothoracic ratio; 57\%). ECG examination showed sinus tachycardia and left atrial (LA) and left ventricular (LV) enlargement. Trans-thoracal echocardiographic (TTE) examination revealed; RWMA: global hypokinetic-akinetic, multi-chamber dilated and severe LV systolic dysfunction (EF 29\%) and diastolic function (Grade IV (E/A Ratio>4)). Valve examination showed moderate-severe MR and moderate TR (TVG $40 \mathrm{mmHg}$ ). Conclusion: Alcohol cardiomyopathy is one of the most common causes of heart failure in young age and carries a poor prognosis if not treated properly. Treatment of alcoholic cardiomyopathy mainly includes total alcohol abstinence along with drugs used to treat systolic heart failure to reverse the condition and correct any nutritional deficiencies.
${ }^{1}$ General Practitioner at Cardiovascular Department BRSU Tabanan Hospital, Bali, Indonesia ${ }^{2}$ Cardiologist at BRSU Tabanan Hospital, Bali, Indonesia ${ }^{3}$ Senior clerkship at Sanglah General Hospital, Bali, Indonesia

*Corresponding to: Gede Wikananda; General Practitioner at Cardiovascular Department BRSU Tabanan Hospital, Bali, Indonesia; g.wikananda@gmail.com

Received: 2019-03-13

Accepted: $2019-07-28$

Published: 2019-08-01

Keywords: alcoholic, cardiomyopathy, Tabanan general hospital

Cite This Article: Wikananda, G., Ariawan, E., Priadnyana, A. 2019. A-41 years old male with alcoholic cardiomyopathy at Tabanan General Hospital, Bali-Indonesia: a case report. Intisari Sains Medis 10 (2): 516-520. D01: 10.15562/ism.v10i2.437

\section{INTRODUCTION}

Alcohol is one of the most frequently consumed beverages in various societies and cultures in the world. The burden caused by alcohol abuse creates losses in health, economic, and social fields. From a health perspective, the complications of excessive alcohol use are both mentally \& physically. They are ranging from physichatric, gastrointestinal, neurological, and cardiological disturbance. In western countries, up to $10 \%$ of the adult population suffers from an alcoholism-related disease. It is among the most common diagnosis requiring hospitalization in North America. The highest prevalence is detected in the third to fifth decade of life. ${ }^{1}$ According to RISKESDAS 2018; national alcohol consumption in Indonesia is 3\%, with predominance by traditional drinks, followed by beer and winery product. Bali is the second province with the most excessive proportion of alcoholic beverages consumption. ${ }^{2}$

Alcoholic cardiomyopathy is a form of cardiac abnormality caused by excessive alcohol consumption. As its name suggests; it mainly affects myocardial structure and function with associated cardiac dysfunction. The clinical picture could be indistinguishable from another form of dilated or familial cardiomyopathies, which is characterized by biventricular dilatation and impaired heart function without any other predisposing etiology. It is suspected that one-third of all cases of nonischemic dilated cardiomyopathy caused by longstanding, excessive alcohol use. ${ }^{1}$

Diagnosis of alcoholic cardiomyopathy is made based on structured anamnesis related to chronic long-standing alcohol abuse. The patient may also complain about cardiac symptoms of heart failure, including; fatigue, shortness of breath, and palpitation, or even asymptomatic at an early stage. ${ }^{1}$

Physical examination could show signs of health 
failure if found on advanced stages, such as fatigue, signs of congestion and associated cardiac murmur. Supporting investigations of cardiac biomarker, laboratories study, electrocardiogram (ECG) and main echocardiography are needed to establish the diagnosis. Early recognition and management based on underlying etiology are important, considering the disease is reversible at an early stage and has a fatal consequence if not appropriately treated. ${ }^{1,3}$ Based on those above, this study aims to determine further the effort of early diagnosis as well as prompt management regarding alcoholic cardiomyopathy as a rare case in Tabanan General Hospital, Bali, Indonesia.

\section{CASE PRESENTATION}

A 41 years old male was found with clinical features of alcoholic cardiomyopathy. $\mathrm{He}$ came to the emergency department unit with the chief complaint shortness of breath since last few days. Shortness of breath was said to emerge on moderate exertion earlier but getting worse over time and progressed to breathlessness at rest. Other complaints were palpitations, fatigue, and long-standing cough. The patient denied chest pain and fever. The patient also denied the history of the same complaint. History of cardiac disease and other chronic diseases was denied by the patient. The patient did not smoke and denied a family history of heart disease. Patient work as office workers and have a sedentary lifestyle. The patient was found to have a history of drinking alcohol in large quantities from his young age. He drinks around six bottles of beer daily and several cups of Arak Bali occasionally since 27 years ago.

Vital sign examination shows; blood pressure was $110 / 70 \mathrm{~mm} \mathrm{Hg}$, pulse was 105 beats/min, and respiratory rate was 26 breaths/min. Physical examination revealed an ill-looking man in shortness of breath and fatigue. Slight edema on both legs was found. From chest auscultation examination, bibasilar crepitation on both lungs was found. On cardiac examination, a systolic murmur of mitral regurgitation (MR) and tricuspid regurgitation (TR) was found.

On supporting examination; complete blood count (CBC) showed leukocytosis $\left(14.7 \times 10^{3} / \mu \mathrm{L}\right)$ with other laboratory values within normal limits. Posterior-anterior chest $x$-ray showed cardiomegaly (cardiothoracic ratio; 57\%). ECG examination showed sinus tachycardia with a left atrial enlargement (LAE) and left ventricular hypertrophy ( $\mathrm{LVH})$.

Trans-thoracal echocardiographic examination was performed after the acute phase has been treated. It revealed regional wall motion abnormality (RWMA); hypokinetic-akinetic, multi-chamber dilated, severe left ventricular (LV) systolic dysfunction (EF 29\%) and diastolic function (Grade IV (E/A Ratio $>4)$ ). Valve examination showed MR moderate-severe and TR moderate (TVG $40 \mathrm{mmHg}$ ). Diagnosis of congestive heart failure (CHF) secondary to alcoholic cardiomyopathy was made. The patient was admitted and given normal saline solution $500 \mathrm{ml}$, oxygen therapy with a nasal cannula, furosemide bolus 2 ampule continued with $40 \mathrm{mg}$ (1-1-0), lisinopril 1 x 2, $5 \mathrm{mg}$, bisoprolol $1 \times 2,5 \mathrm{mg}$ and letonal $1 \times 25 \mathrm{mg}$ as pharmacologic treatment.

\section{DISCUSSION}

Excessive alcohol consumption induces several pathologic changes in the myocardial structure. It shares indistinguishable feature from other forms of dilated or familial cardiomyopathy. The exact pathogenesis is still unclear, but it is believed to be related to direct toxic result of ethanol or its metabolites, which results in altered metabolism of fatty acid, disruption of cardiac calcium hemostasis, loss of contractile protein, decreased myocardial protein synthesis and impaired mitochondrial function. Histologically, it features myofibrillar necrosis and fibrosis. Various studies have also shown that alcohol exerts adverse inotropic effects on the myocardium. Ethanol may also cause oxidative stress through the generation of free radicals. It also increases circulating catecholamine. This condition is believed to play a role in generating myocardial damage, which can be proven by the increase of troponin release. ${ }^{1,4}$

Genetics factor is also assumed to contribute in developing alcohol cardiomyopathy. Studies showed that not all individuals with chronic alcohol abuse develop the same conditions. Individuals with the angiotensin-converting enzyme (ACE) DD genotype tend to have an increased risk of developing alcoholic cardiomyopathy, ${ }^{1,3,5}$ Total alcohol abstinence combined with drugs used in systolic heart failure and corrected any nutritional deficiencies is the mainstay of the treatment. ${ }^{2}$

This patient clinical finding matched with alcoholic cardiomyopathy. From anamnesis, the patient complained shortness of breath, which gets worse over time. These complain is accompanied by long-standing cough and palpitation. Physical examination revealed bibasilar crepitation on the chest and slight edema on both legs. From this examination, we can suspect that the patient was in an acute state of heart failure (HF). Supporting examination with $\mathrm{x}$-ray showed cardiomegaly, which is usually a sign of another pathologic condition and reinforces our suspicion to HF. Echocardiography examination confirmed that 


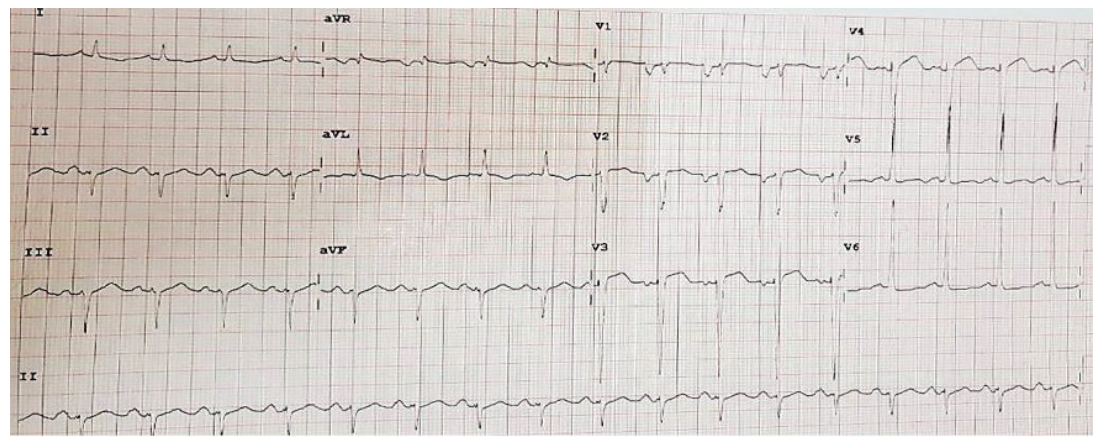

Figure 1. ECG showing sinus tachycardia, LAE and LVH

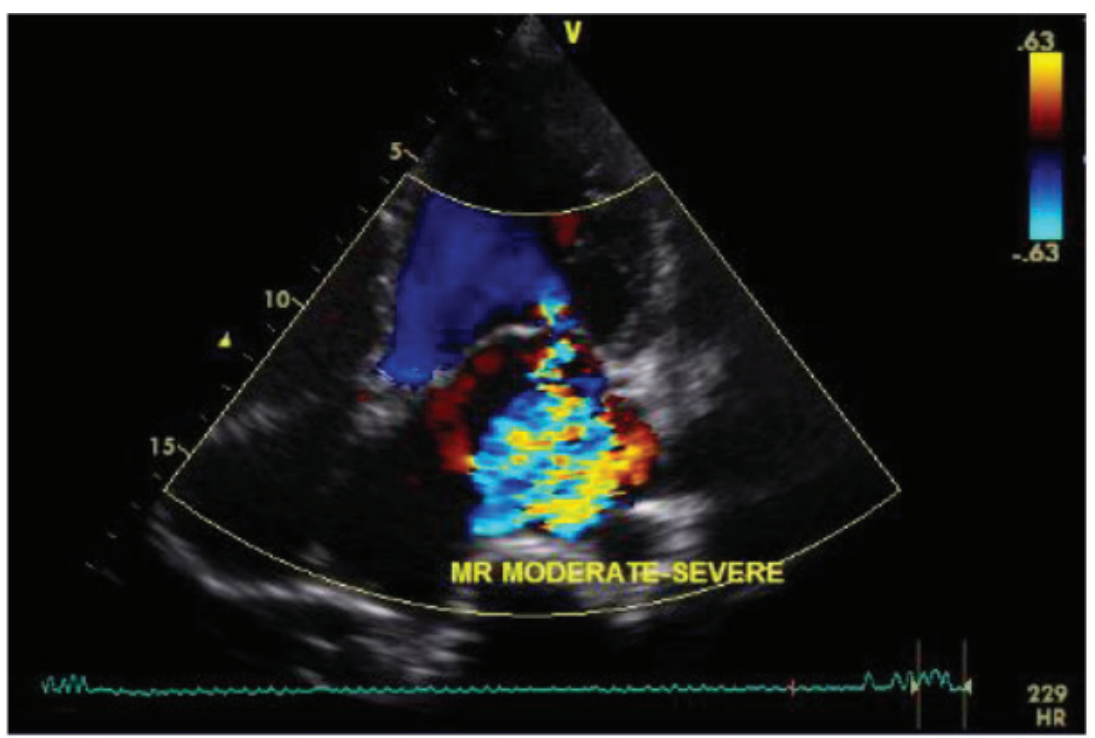

Figure 2. Flow Across Mitral Valve Showing Mitral Regurgitation

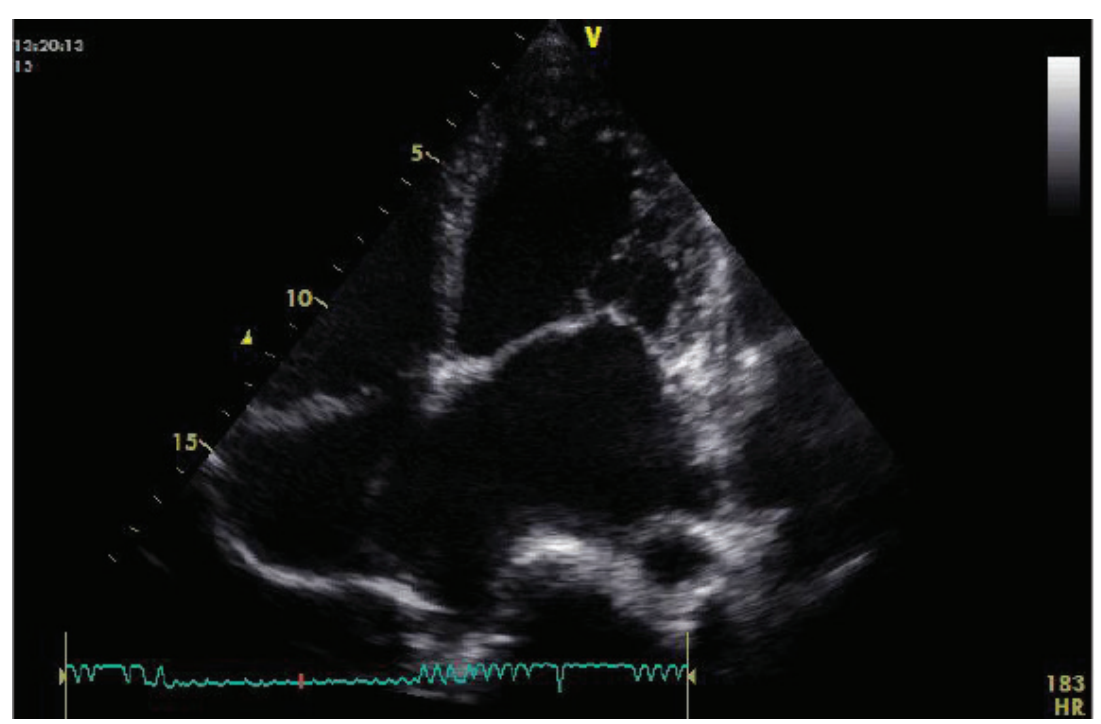

Figure 3. Parasternal Long Axis (PLAX) showing multi-chamber dilation, a characteristic of alcoholic cardiomyopathy dilated cardiomyopathy was the underlying mechanism for patient complaints. It is a very useful supporting examination to help exclude other nonischemic cardiomyopathies. Without evidence of previous heart disease nor chronic diseases, anamnesis is very important to explore risk factors related to patient condition to aid in confirming the diagnosis. ${ }^{3}$ From this patient, chronic, longstanding alcohol ingestion from his young age without any other predisposing factors is one of the most reliable clues leads to a diagnosis of alcoholic cardiomyopathy. ${ }^{6}$

Mild reduction in cardiac functions occurs in chronic alcoholics before symptoms appear. On later stages, HF symptoms could occur due to severe abnormalities on systolic and diastolic function. The symptoms that appear ranges from progressive exercise limitation to acute fulminant heart failure, in the setting of multi-chamber dilation and hypokinetic contraction. This condition can be evaluated by Doppler echocardiographic examination. This patient had a severe reduction on both systolic (EF 29\%) and diastolic functions (Grade IV (E/A Ratio $>4$ ) which is enough to explain the patient condition during physical examination.

Heart rhythm abnormalities/ arrhythmias are other common findings in the setting of alcoholic cardiomyopathy, which affect two-thirds of patients. It is characterized by palpitation or feeling of discomfort in the chest and abnormalities on cardiac rhythm on ECG examination. ${ }^{6}$ The etiology has been speculated to be the result of a variety of electrical abnormalities. It is believed that an arrhythmogenic substrate is resulting from myocardial fibrosis leading to an irritable focus that is easily triggered. Other hypotheses include stretching of myocardial fibers induced by increased left ventricular end diastolic volume (LVEDV) leading to an arrhythmic substrate. However, Jeffrey et al. stated that the disruption of the link between the sarcolemma, cytoskeleton and the sarcomere had been shown to be associated with the disease, with disturbance in ion channel function as the final common pathway associated with rhythm disorders. ${ }^{7}$

Functional mitral valve regurgitation is highly prevalent among patients with dilated cardiomyopathy as a result of the geometric and hemodynamic change. ${ }^{8,9}$ It is associated with high morbidity and mortality. Functional MR in the setting of dilated cardiomyopathy mainly occurs because of restricted mitral valve leaflet motion. According to Carpentier classification, this condition arises in the absence of anatomic leaflet, papillary muscle, and chordal abnormalities. ${ }^{10}$ The degree of functional MR is determined by the 
mitral valve (MV) and LV characteristic. From MV characteristic, it arises from changes in mitral annular function and size. The MV annulus is flattened and demonstrates reduced circumferential contraction and intercommisural folding. From LV characteristic, increased LV sphericity or remodeling of posterior and inferior LV walls will cause papillary muscle displacement which contributes to MV leaflet tethering. ${ }^{10}$

The other most important LV characteristic is longitudinal contractility (a strain of LV-mid lateral wall) and intra LV dyssynchrony. ${ }^{9,10}$ Intra LV dyssynchrony is associated with a mechanical activation time delay between LV segments adjacent to the papillary muscles. Kanazaki et al.suggest that MR was related to a systolic imbalance of forces acting on the papillary muscles due to uncoordinated regional LV activation in these segments, causing geometric changes in the mitral leaflet attachments. ${ }^{11}$ The mechanism of functional TR in alcoholic cardiomyopathy is believed to quite similar to MR which is related to abnormal heart muscle condition, ventricular geometric, and hemodynamic change.

Early management and total alcohol abstinence is the mainstay of therapy to prevent disease progression, relieve symptoms, and even reversal of disease before fibrosis sets in. The prognosis is very poor with the continued use of alcohol, with a mortality rate as high as $42 \%$ at three years. Based on the study, LV function improvement could be seen on 6 months after total alcohol abstinence and proper medical therapy; whereas complete recovery could be achieved approximately in 18 months. ${ }^{4}$ Treatment of alcoholic cardiomyopathy follows the usual regiment for treatment of systolic heart failure. This including vasodilator, diuretics, angiotensin converting enzyme (ACE) inhibitor, beta blocker, and digitalis for rate control when appropriate. ${ }^{5}$ Other therapeutic strategies depending on complications may be needed. The patient was given above regiment to relieve his sign \& symptoms. Vasodilator therapy has benefit in decreasing ventricular filling pressure, systemic vascular resistance (SVR), and ventricular volumes. Diuretics agent has an interest in reducing volume overload then chamber pressure. Meanwhile, ACE inhibitor appears to be related to vasodilatation and as an anti-remodeling agent. Last but not least, beta blocker mitigating LV hypertrophy, remodeling, lowering filling pressure, and improve contractility.

In selected patients, cardiac resynchronization therapy (CRT) and surgical intervention (valve repair, replacement, ventricular reconstruction or cardiac transplantation) may needs when medical treatment for left ventricular dysfunction and other complication when exist has been inadequate. CRT has shown to reduce functional MR significantly, by targeting myocardial dyssynchrony. ${ }^{10} \mathrm{CRT}$ also has been demonstrated to substantially reduce functional MR in conjunction with ACE-inhibitor and beta-blocker therapy. This appears related to activation at the papillary muscle insertion sites. Referral to a psychiatrist may need to deal with treatment associated with alcohol addiction because abstain from drinking alcohol is one of the main components of alcoholic cardiomyopathy treatment.

\section{CONCLUSION}

Alcoholic cardiomyopathy is a condition caused by excessive alcohol consumption, which causes a reduction in cardiac function and disturbance in heart pumping ability. Clinical manifestation ranges widely, from asymptomatic to acute fulminant heart failure, depending on the degree of deterioration of heart function. Early diagnosis, total alcohol abstinence and early management with drugs used in systolic heart failure are the mainstay of treatment to prevent disease progression, relieve symptoms, and prevent complications and reversal of the disease if possible.

\section{CONFLICT OF INTEREST}

The authors declare that there is no competing interest regarding the manuscript

\section{FUNDING}

The authors are responsible for the funding of study without the involvement of grant, scholarship, or any other resources of funding

\section{AUTHOR CONTRIBUTION}

All of the authors are equally contributed to the study from the cases framework, data gathering, data analysis, until reporting the result of study.

\section{REFERENCES}

1. Uchenna DI, Anyalechi JI, Jesuorobo DE. Alcoholic Cardiomyopathy in A 39 Year Old Female: A Case Report. The Internet Journal of Cardiology. 2010; 10(1):1-5

2. Balitbang Kemenkes RI. Tingkat Konsumsi Alkohol. Riset Kesehatan Dasar; RISKESDAS. Jakarta: Balitbang Kemenkes RI. 2018

3. Guzzo-Merello G, Cobo-Marcos M, Gallego-Delgado M, Garcia-Pavia P. Alcoholic cardiomyopathy. World J Cardiol. 2014;6(8):771-81

4. Mahmoud S, Beauchesne LM, Davis DR, Glover C. Acute reversible left ventricular dysfunction secondary to alcohol. Can J Cardiol. 2007;23(6):475-477

5. Johnson BA, Addolorato G, Leggio L, d'Angelo C, et al. Physical considerations for treatment complications 
of alcohol and drug use and misuse. In: Johnson BA ed Addiction medicine. Springer Science and Business Media, LLC. 2011;1115-45

6. Maisch B. Alcoholic cardiomyopathy, the result of dosage and individual predisposition. Herz 2016.41(6):484-493

7. Towbin, Jeffrey A., Lorts, Angela. Arrhythmias and Dilated Cardiomyopathy. Journal of the american college of cardiology. 2011;57(21):2169-71

8. Mumtaz A, Setiawan A. Faktor resiko kardiomiopati dilatasi di rumah sakit dr. Kariadi Semarang. Jurnal Kedokteran Diponegoro. 2017;6(1);20-27

9. Donal E, De Place C, Kervio G, Bauer F, Gervais R, Leclerce C, et al. Mitral regurgitation in dilated cardiomyopathy: value of both regional left ventricular contractility and dyssynchrony. Eur J Echocardiogr. 2009;10(1):133-8.

10. Punnose L, Burkhoff D, Cunningham L, Horn EM. Functional mitral regurgitation: therapeutic strategies for a ventricular disease. J Card Fail. 2014; 20(4):252-67.
11. Kanzaki H, Bazaz R, Schwartzman D, Dohi K, Sade LE, Gorcsan J 3rd. A mechanism for immediate reduction in mitral regurgutation after cardiac resynchronization therapy: insights from mechanical activation strain mapping, J Am Coll Cardiol. 2004;44:1619-1625

12. Mojadidi, Mohammad K, Zaman Muhammad O, Elgendy Islam Y, et al. Patent foramen ovale closure: What are the Indications? A summary of lessons learned from positive randomized trial data. Cardiac Interventions Today. 2018.12(3);28-33

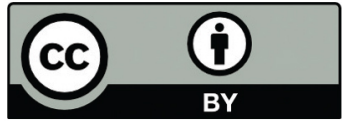

This work is licensed under a Creative Commons Attribution 